\title{
BMJ Open Prevalence and prescription patterns of oral glucocorticoids in adults: a retrospective cross-sectional and cohort analysis in France
}

Anne Bénard-Laribière, ${ }^{1}$ Antoine Pariente, ${ }^{1,2}$ Elodie Pambrun, ${ }^{1}$ Bernard Bégaud, ${ }^{1,2}$ Laurence Fardet, ${ }^{3,4}$ Pernelle Noize $e^{1,2,5}$

To cite: Bénard-Laribière $A$, Pariente A, Pambrun E, et al. Prevalence and prescription patterns of oral glucocorticoids in adults: a retrospective cross-sectional and cohort analysis in France. BMJ Open 2017;7:e015905. doi:10.1136/ bmjopen-2017-015905

- Prepublication history and additional material for this paper are available online. To view these files please visit the journal online (http://dx.doi. org/10.1136/bmjopen-2017015905).

Received 9 January 2017 Revised 27 March 2017 Accepted 10 April 2017

\section{CrossMark}

${ }^{1}$ University of Bordeaux, Inserm, Bordeaux Population Health Research Center, Pharmacoepidemiology Team, Bordeaux, France

${ }^{2} \mathrm{CHU}$ Bordeaux, Service de Pharmacologie Médicale, Bordeaux, France ${ }^{3}$ Université Paris Est Créteil (UPEC), Créteil, France

${ }^{4} \mathrm{CHU}$ Henri Mondor, Service de Dermatologie, Créteil, France ${ }^{5} \mathrm{CIC}$ Bordeaux CIC1401, Bordeaux, France

Correspondence to Anne Bénard-Laribière; anne.benard@u-bordeaux.fr

\section{ABSTRACT}

Objectives To study trends in use of oral glucocorticoids (GCs) among adults, characteristics of oral GC initiators and prescriptions for the prevention of potential adverse effects associated with GC therapy.

Design First, a cross-sectional study repeated yearly was performed from 2007 to 2014 in a nationwide representative sample. Second, characteristics of initiators and patterns of GC therapy during the year following treatment initiation were described in a cohort of patients who began GC between 2007 and 2013.

Setting Population-based study using data from the French reimbursement healthcare system (covering approximately $90 \%$ of the population) in patients aged $\geq 18$ years.

Results Over the study period, the prevalence of oral GC use ranged from $14.7 \%$ to $17.1 \%(95 \% \mathrm{Cl} 17.0 \%-$ $17.2 \%)$ with a significant increase of $14.1 \%(95 \% \mathrm{Cl}$ $+13.5 \%$ to $+14.8 \%)$. The $2007-2013$ cohort of oral GC initiators comprised 206759 individuals. Oral GC use was mostly short-term ( $68 \%$ of unique reimbursement) and more than half of short-term users took concurrent antibiotics or respiratory/otological drugs. Chronic users ( $\geq 6$ reimbursements/year) represented $1.8 \%(n=3789)$ of the cohort. The proportion of chronic users with comorbidities likely to be worsened by GC use (diabetes, psychotic disorders, osteoporosis) was $25 \%$. Among patients at increased risk of osteoporosis, $62 \%$ received specific prevention/monitoring measures and only $27 \%$ had a bisphosphonate. Half of chronic oral GC users had a concurrent reimbursement of a proton pump inhibitor in the absence of non-steroidal anti-inflammatory drug use. Conclusions Oral GC use was highly widespread and increased among adults from 2007 to 2014. The overwhelming short-term use could mainly concern a growing use of unjustified prescriptions rather than situations with a favourable benefit/risk ratio. For chronic users, our findings plead for the development of interventions designed to improve monitoring with regard to the frequent comorbidities at risk and inappropriate prescribing of preventive therapeutic measures.

\section{INTRODUCTION}

Oral glucocorticoids (GCs) have been used for more than 60 years for their substantial
Strengths and limitations of this study

- The main strength is that it is representative of the general population owing to the data source, a healthcare insurance database with exhaustive recording of reimbursements and hospitalisations.

- A limitation is that the database does not include direct information on medical indications, which could be derived from data on chronic diseases, hospitalisations and concurrent drugs.

- Altogether, this population-based study provides a description of oral glucocorticoid use and trends in adults over an 8-year period from 2007 to 2014.

anti-inflammatory and immunosuppressive effects in several acute and chronic disorders, both for reducing disease activity and pain. ${ }^{1}$ However, their use is limited by the occurrence of adverse reactions related to their pharmacological properties that are mainly to be feared with higher dosages or long-term use. These associated risks include infections, osteoporosis and fractures, hyperglycaemia, neuropsychiatric disorders and muscle atrophy. Recommendations on the management of GC therapy based on expert consensus are available for the prevention of GC-induced osteoporosis $^{2-4}$; regarding other significant adverse reactions, advice on pretreatment and treatment monitoring have been issued ${ }^{56}$ but no consensual recommendations exist.

Besides these well-known adverse consequences, the relevance of some other potential adverse reactions is debated, such as the impact on electrolyte homeostasis due to mineralocorticoid effects or the risk of peptic ulcer. While potassium loss seems negligible in practice, ${ }^{7}$ some physicians persist in prescribing potassium supplementation, which in some situations may carry a risk of marked hyperkalaemia. ${ }^{8}$ Similarly, despite the literature suggesting no benefit from proton pump 
inhibitors (PPIs) prophylaxis in patients taking systemic GCs without concomitant non-steroidal anti-inflammatory drug (NSAID) use, ${ }^{910}$ many prescribers still consider GCs as a cause of upper gastrointestinal complications and systematically add PPIs to their prescriptions. ${ }^{11}$

Few studies have reported the use of oral GCs in the general population, and short-term use has rarely been quantified as it is considered safe. This population-based study aimed at describing trends in the use of oral GCs among adults, the characteristics of GC initiators and the prescriptions for the prevention of potential adverse effects associated with GC therapy.

\section{METHODS}

\section{Data source}

The study was conducted using the French reimbursement database (Echantillon Généraliste de Bénéficiaires, EGB). The EGB is a representative sample of the population covered by the national healthcare insurance system (approximately 90\% of the whole population, irrespective of socioeconomic status) obtained by $1 / 97$ th random sampling with stratification on sex and age. For all beneficiaries, it consists of the exhaustive recording of drug reimbursements, with identification of medication packs, including the number and dosage strengths of treatment units. The database also contains hospitalisation data (diagnoses and dates) and the existence of certain chronic diseases (Affections de Longue Durée, ALD, an administrative status allowing full reimbursement of healthcare for a given condition, eg, diabetes, cancer, psychosis). Diagnoses or indications for prescribing are not collected in the EGB database, nor the dose prescribed or the duration of treatment. Details on the EGB database have been described elsewhere. ${ }^{12} 13$

\section{Study design}

Cross-sectional study

In order to study temporal trends in the use of GCs, a cross-sectional study was repeated yearly among the population aged $\geq 18$ years from 1 January 2007 to 31 December 2014. All individuals who had at least one reimbursement of an oral GC (ie, betamethasone, dexamethasone, methylprednisolone, prednisolone and prednisone) were identified for each year studied.

\section{Cohort study}

To study characteristics of GC users and therapeutic behaviour associated with the prescription, a cohort of oral GC initiators was identified. GC initiators were defined as an incident reimbursement of oral GC between 1 January 2007 and 31 December 2013, without any in the preceding year. This definition was retained to ensure incident use was identified in a conservative manner even if other definitions can be found in the literature (eg, prescription-free, 90 -day ${ }^{14}$ or 6 -month period $\left.^{1516}\right)$. The index date was the date the incident GC was reimbursed. Each GC initiator was followed until 1 year since index date, the date of death or the end of data availability in the database, whichever came first. Identified individuals could only contribute once to the cohort constitution.

\section{Characteristics of GC initiators}

GC initiators were described in terms of age and sex at index date. Comorbidities that may represent situations at risk in the event of GC use (ie, diabetes, psychotic disorders and osteoporosis) were described, as were chronic disorders constituting recognised indications for GC therapy: rheumatic diseases (eg, rheumatoid arthritis, polymyalgia rheumatica/giant cell arteritis, lupus and vasculitis), obstructive pulmonary diseases (ie, asthma, chronic obstructive pulmonary disease and chronic respiratory failure), inflammatory bowel diseases (ie, Crohn's disease and ulcerative colitis) and multiple sclerosis. Comorbidities and indications for oral GC treatment were identified using data from diagnoses related to hospital stays or chronic diseases (ALDs) and medication reimbursement data in the 12-month period preceding the patient's index date. A description of drugs reimbursed at index date (concurrent drugs) was also performed as these potentially reflect the indication of GC therapy.

\section{Therapeutic behaviour associated with the prescription of GCs}

Over the year following GC treatment initiation, we scrutinised two types of preventive measures: (1) those that should be systematically considered, such as prevention/monitoring of osteoporosis among individuals at increased risk of osteoporosis; and (2) those for whom no consensus exists and/or that might be inappropriate (potassium supplementation without serum potassium assay, and PPI prophylaxis without concurrent NSAID or aspirin use).

To assess the prevention and monitoring of osteoporosis, individuals at increased risk of GC-induced osteoporosis were defined as those who had at least six reimbursements of GCs during the 12-month period following the index date and (1) were aged 70 years and older or (2) had a history of untreated osteoporosis during the 12 months preceding the index date. Measures for prevention/monitoring of osteoporosis among these individuals were identified by at least one of the following criteria: (1) bone mineral density measurement (at least one reimbursement for dual-energy X-ray absorptiometry, DXA) or (2) prescription of drugs indicated for osteoporosis management (at least one reimbursement for calcium, vitamin $\mathrm{D}$, bisphosphonates, denosumab, raloxifene, teriparatide, strontium ranelate and calcitonin).

For non-consensually recommended measures, potentially inappropriate potassium supplementation was defined as at least one concurrent reimbursement of oral GC and potassium supplements without any serum potassium assay during the two preceding weeks. A priori non-indicated prescription of PPIs was defined as at least one concurrent reimbursement of oral GC and PPI in the absence of NSAID or aspirin on the same date. 


\section{Data analysis}

Trends in use of oral GCs

The annual prevalence of GC use was defined as the proportion of GC users per 100 individuals for the corresponding year. It was first calculated for GCs overall and then by considering each GC individually. All prevalence estimates were further stratified according to the number of GCs reimbursed per year $(1,2-5, \geq 6$ ) and by sex and age (five categories according to age on 1 January in each year: 18-49 years, 50-59 years, 60-69 years, 70-79 years and $\geq 80$ years), and were quantified together with their two-sided $95 \%$ CIs. To study trends in prevalent use over the study period, relative changes in prevalence of use were estimated by using the year 2007 as reference. Relative change estimates, quantified together with their two-sided $95 \%$ CIs, were calculated using the percentiles bootstrap method.

\section{Characteristics of GC initiators and therapeutic behaviour} associated with prescription of GCs

All parameters were examined overall and stratified according to the duration of therapy. The EGB database does not provide the total duration of treatments, but GC treatment is issued for a maximum of 30 days in France and individuals have to renew their treatment each month. We consequently assessed GC treatment duration according to the number of oral GC reimbursements (consecutive or not) identified during the 12-month period following the index date. Users who had a unique reimbursement were arbitrarily defined as short-term users, those who had 2-5 reimbursements as mid-term users and those with $\geq 6$ reimbursements as long-term users. We assumed that individuals with $\geq 6$ reimbursements/year were treated for chronic diseases. Measures for the prevention of osteoporosis were examined only for individuals with an increased risk of osteoporosis as defined above.

All analyses were performed using SAS V.9.4 software. All codes used for the identification of the studied comorbidities and medications are listed in online supplementary tables 1-5.

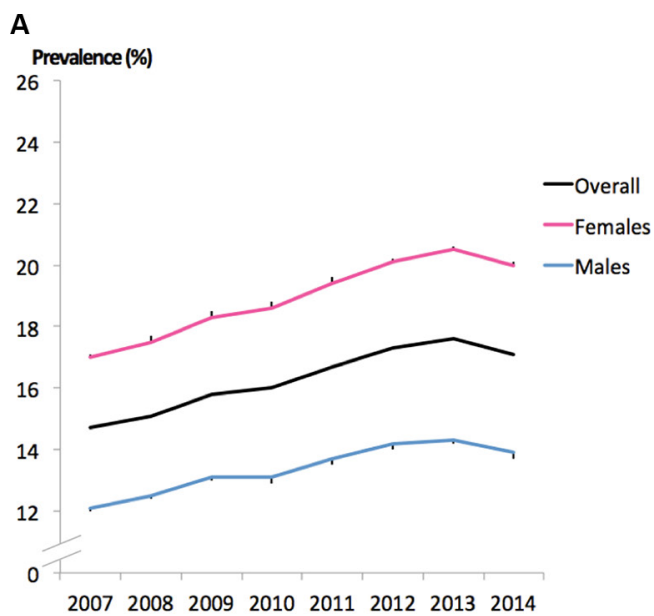

RESULTS

\section{Trends in use of oral GCs from 2007 to 2014}

Of the 382572 individuals included in the study in 2007, 56126 had at least one reimbursement of an oral GC: the prevalence of GC use was $14.7 \% \quad(95 \%$ CI $14.6 \%$ to $14.8 \%$ ) in 2007 . It was $17.1 \%$ (95\% CI $17.0 \%$ to $17.2 \%$ ) in 2014 , corresponding to a $14.1 \%$ increase $(95 \% \mathrm{CI}+13.5 \%$ to $+14.8 \%)$ compared with 2007 (figure 1). This rise was more pronounced in individuals aged 50-59 years $(+18.4 \%(95 \% \mathrm{CI}+17.0 \%$ to $+20.0 \%))$ and $60-69$ years $(+19.7 \%(95 \% \mathrm{CI}+17.9 \%$ to $+21.5 \%))$. It mostly concerned prednisolone $(+21.6 \% \quad(95 \%$ CI $+20.8 \%$ to $+22.3 \%))$ (see online supplementary figure $\mathrm{S} 1$ ); this was the most used GC over the study period, irrespective of age and sex. The prevalence of use was higher among women whatever their age, the highest value being observed in those aged $50-59$ years $(21.9 \%$ in 2014 (95\% CI $21.4 \%$ to $22.3 \%)$ ) (see online supplementary figure S2). Concerning the number of GCs reimbursed per year, the prevalence of unique reimbursement slightly increased from $10.3 \%$ in 2007 to $11.8 \%$ in $2014(+12.7 \%$ (95\% CI $+11.8 \%$ to $+13.5 \%))$. The proportion of individuals who had 2-5 reimbursements per year rose from $3.8 \%$ to $4.6 \% \quad(+18.6 \%$ $(95 \% \mathrm{CI}+17.4 \%$ to $+19.9 \%))$. Conversely, the percentage of individuals with $\geq 6$ reimbursements per year remained stable and ranged between $0.6 \%$ and $0.7 \%(+7.9 \%$ increase $(95 \%$ CI $+3.8 \%$ to $+11.8 \%)$ compared with 2007$)$.

\section{Characteristics of GC initiators}

The 2007-2013 cohort of GC initiators comprised 206759 individuals; $58.0 \%$ were women and the median age was 45 years (IQR: 32-59). More than two-thirds of initiators $(67.6 \%)$ had a unique reimbursement of GC over the year following treatment initiation (short-term users). Midterm users represented $30.6 \%$ of the study cohort and long-term users $1.8 \%$. Compared with short-term and mid-term users, long-term users were more likely to be older (median age: 63 years, IQR: $49-76)$; one-quarter $(24.5 \%)$ had at least one comorbidity at treatment initiation that was likely to increase the risk of adverse drug reaction in the event of GC

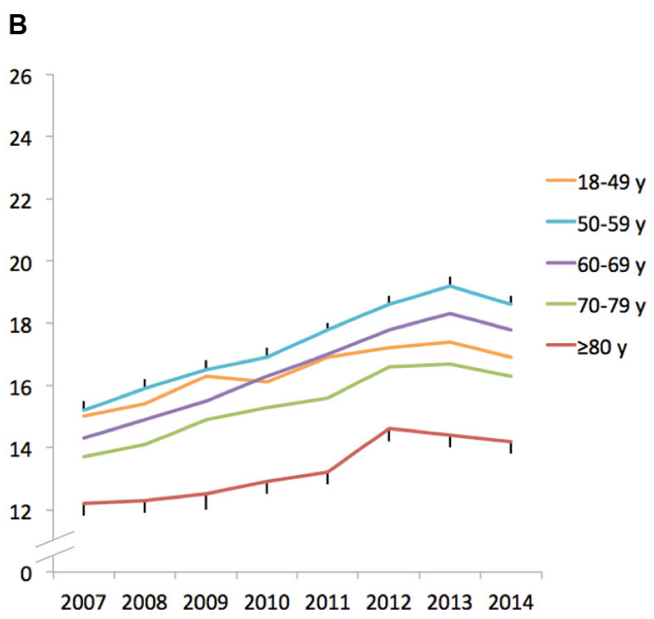

20072008200920102011201220132014

Figure 1 Trends in prevalence of oral glucocorticoid use in France per year from 2007 to 2014. Prevalence estimates with 95\% Cls (error bars) (A) overall and by sex, and (B) by age. 
Table 1 Characteristics of oral glucocorticoid (GC) initiators, overall and according to the number of oral GC reimbursements over the year following treatment initiation (figures are percentages)

\begin{tabular}{|c|c|c|c|c|}
\hline & All GC initiators & Short-term users* & $\begin{array}{l}\text { Mid-term } \\
\text { users* }\end{array}$ & $\begin{array}{l}\text { Long-term } \\
\text { users* }\end{array}$ \\
\hline & $n=206759$ & $n=139703$ & $n=63267$ & $n=3789$ \\
\hline Men & 42.0 & 43.3 & 39.1 & 44.4 \\
\hline \multicolumn{5}{|l|}{ Age groups (years) } \\
\hline $18-49$ & 58.8 & 60.0 & 58.2 & 25.5 \\
\hline $50-59$ & 16.6 & 16.3 & 17.2 & 16.9 \\
\hline $60-69$ & 12.0 & 11.6 & 12.3 & 18.9 \\
\hline $70-79$ & 7.8 & 7.4 & 7.7 & 22.0 \\
\hline$\geq 80$ & 4.9 & 4.8 & 4.6 & 16.7 \\
\hline Mean number of reimbursements/year $( \pm S D)$ & $1.6( \pm 1.4)$ & $1^{*}$ & $2.5( \pm 0.8)$ & $9.2( \pm 3.1)$ \\
\hline Comorbidities at risk for GC users $\dagger$ & 10.6 & 10.0 & 10.9 & 24.5 \\
\hline Diabetes & 5.3 & 5.2 & 5.1 & 12.1 \\
\hline Psychotic disorders & 2.6 & 2.5 & 2.9 & 3.6 \\
\hline Osteoporosis & 3.3 & 3.0 & 3.6 & 11.0 \\
\hline Identified GC recognised indications $†$ & 27.3 & 23.7 & 33.4 & 61.1 \\
\hline Obstructive pulmonary diseases & 21.3 & 19.1 & 26.0 & 26.2 \\
\hline Cancer & 6.4 & 4.9 & 8.2 & 31.9 \\
\hline Rheumatic diseases & 1.0 & 0.6 & 1.1 & 12.1 \\
\hline Rheumatoid arthritis & 0.4 & 0.2 & 0.5 & 5.7 \\
\hline Polymyalgia rheumatica/giant cell arteritis & 0.1 & 0.0 & 0.1 & 3.9 \\
\hline Inflammatory bowel diseases & 0.6 & 0.4 & 0.9 & 3.3 \\
\hline Multiple sclerosis & 0.2 & 0.2 & 0.2 & 0.2 \\
\hline \multicolumn{5}{|l|}{ Concurrent drugs at index date } \\
\hline Antibiotics & 59.1 & 60.6 & 57.7 & 23.9 \\
\hline Respiratory/otological drugs $\ddagger$ & 50.1 & 51.1 & 49.9 & 16.7 \\
\hline $\begin{array}{l}\text { Concurrent antibiotics and respiratory/ } \\
\text { otological drugs }\end{array}$ & 31.8 & 32.3 & 32.2 & 8.9 \\
\hline Anti-inflammatory & 6.5 & 6.4 & 6.9 & 7.2 \\
\hline Analgesics & 46.0 & 46.5 & 45.6 & 34.6 \\
\hline
\end{tabular}

*Short-term users: one reimbursement/year; mid-term users: 2-5 reimbursements/year; long-term users: $\geq 6$ reimbursements. †At least one comorbiditie or indication.

$\ddagger$ Nasal and throat preparations, antihistamines for systemic use, cough and cold preparations, and otological drugs.

use (diabetes: 12.1\%; osteoporosis: $11.0 \%$; psychotic disorders: $3.6 \%)$. Recognised GC indications were identified in $61.1 \%$ of long-term users. Among these potential indications, obstructive pulmonary diseases $(26.2 \%)$, rheumatic diseases $(12.1 \%)$ and inflammatory bowel diseases $(3.3 \%)$ were the most frequent, and nearly $32 \%$ of these individuals had a cancer (table 1).

Among all GC users, concurrent antibiotics (59.1\%), respiratory/otological drugs $(50.1 \%)$ or both $(31.8 \%)$ were frequently reimbursed at the index date, suggesting that underlying ENT (ear, nose, throat) and upper respiratory tract infections were often present (table 1).

\section{Therapeutic behaviour associated with prescription of GCs}

Among GC initiators, 1469 (0.7\%) individuals were considered at increased risk of GC-induced osteoporosis related to long-term treatment ( $\geq 6$ reimbursements/ year) and to age ( $\geq 70$ years) or to a history of non-treated osteoporosis. Among them, $61.5 \%$ had at least one measure aiming at preventing/monitoring osteoporosis over the year following treatment initiation: DXA was performed in $189(12.9 \%)$ individuals and 891 $(60.6 \%)$ individuals were reimbursed at least one drug for osteoporosis management. Nearly $55 \%$ of at-risk individuals received calcium and/or vitamin $\mathrm{D}, 27.4 \%$ a bisphosphonate and $5.0 \%$ another drug for osteoporosis prevention (table 2 ).

Over the year following treatment initiation, 10.8\% of GC initiators had at least one concurrent reimbursement of oral GC and PPI without known concurrent NSAID or aspirin use; this concerned nearly half $(49.8 \%)$ of long-term users versus $7.1 \%$ of shortterm users. Concurrent reimbursement of oral GCs 
Table 2 Measures for prevention/monitoring of osteoporosis among individuals at increased risk (figures are percentages)

Individuals at increased risk* $\mathrm{n}=1469$

\begin{tabular}{lc}
\hline $\begin{array}{l}\text { At least one measure during the year } \\
\text { following treatment initiation }\end{array}$ & 61.5 \\
\hline $\begin{array}{l}\text { Dual-energy X-ray absorptiometry } \\
\text { Drugs for osteoporosis management }\end{array}$ & 12.9 \\
\hline Vitamin D \pm calcium & 60.6 \\
\hline Bisphosphonates & 54.2 \\
\hline Bisphosphonates & 27.4 \\
\hline $\begin{array}{l}\text { Fixed association of } \\
\text { bisphosphonates and vitamin D } \pm\end{array}$ & 20.8 \\
$\quad$ calcium & 9.1 \\
\hline $\begin{array}{l}\text { Other drugs for osteoporosis } \\
\text { management }\end{array}$ & 5.0 \\
\hline Calcitonin & 0.7 \\
\hline Denosumab & 0.3 \\
\hline Raloxifene & 0.6 \\
\hline Strontium ranelate & 3.1 \\
\hline Teriparatide & 0.6 \\
\hline
\end{tabular}

${ }^{*}$ At least six reimbursements of oral glucocorticoids per year and (1) age $\geq 70$ years or (2) history of untreated osteoporosis.

and potassium supplementation concerned $23.7 \%$ of long-term users, of whom $37.3 \%$ never had any serum potassium assay during the 2 weeks preceding the prescription. Conversely, concurrent use of oral GCs and potassium supplementation was infrequent among individuals who had $<6$ reimbursements of GCs over the year following treatment initiation $(<2 \%)$ (table 3$)$.

\section{DISCUSSION}

\section{Statement of principal findings}

This population-based study provides a representative description of oral GC use in adults and its trends over the past 7 years in France. The annual prevalence of GC use in the general population, which was already high in 2007 , increased by $14 \%$, that is, about $2 \%$ per year. In 2014, 17\% of the French adult population had at least one reimbursement of an oral GC. The overwhelming majority (68\%) of new GC use was short-term (unique reimbursement) and apparently related to ENT and upper respiratory tract infections. Overall, 1.8\% of GC initiators were considered as chronic users. Of note, comorbidities likely to be worsened by GC use (diabetes, psychotic disorders, osteoporosis) were found at treatment initiation among nearly one-quarter of chronic users. Therapeutic measures for the prevention of GC-induced osteoporosis appeared to be insufficiently prescribed among individuals judged at increased risk. Conversely, the concurrent prescription of PPIs and potassium supplementation was found to be frequent, in particular in chronic users, although the toxicity of GCs for the upper gastrointestinal tract and the risk of hypokalaemia are questionable or, at least, debated.

\section{Strengths and weaknesses of study}

The major strength of the study stands in the use of the EGB database, fully representative of the whole French population, which ensures the generalisability of the results to a national level. This use however implies some limitations inherent to almost all studies conducted on reimbursement claims databases. First, the database does not provide direct information about medical indication for each reimbursement, so we used data from diagnoses related to hospital stays or chronic diseases and concurrent drugs as proxies of potential GC indications. Second, given that the database does not provide the prescribed duration of treatments, this was defined according to the number of reimbursements per year. If a unique dispensing appears as an indisputable indicator of short-term use, we postulated that individuals with at least six reimbursements per year were chronic users even if renewals were not consecutive, which can be discussed.

\section{Strengths and weaknesses in relation to other studies}

The use of oral GCs in the general population has received little attention until now. ${ }^{17-21}$ Contrary to the present study, none of the previous studies were truly

Table 3 Measures for kalaemia and gastric protection associated with the prescription of oral GC therapy over the year following treatment start (figures are percentages)

\begin{tabular}{|c|c|c|c|c|}
\hline & $\begin{array}{l}\text { All GC initiators } \\
n=206759\end{array}$ & $\begin{array}{l}\text { Short-term users* } \\
n=139703\end{array}$ & $\begin{array}{l}\text { Mid-term users* } \\
\mathrm{n}=63267\end{array}$ & $\begin{array}{l}\text { Long-term users* } \\
\mathrm{n}=\mathbf{3 7 8 9}\end{array}$ \\
\hline $\begin{array}{l}\text { At least one concurrent reimbursement of } \\
\text { GC and potassium supplements }\end{array}$ & 1.3 & 0.5 & 1.8 & 23.7 \\
\hline $\begin{array}{l}\text { Without any serum potassium level } \\
\text { measurement during the preceding } \\
\text { 2-week period }\end{array}$ & 0.8 & 0.4 & 1.3 & 8.8 \\
\hline
\end{tabular}

*Short-term users: one reimbursement/year; mid-term users: 2-5 reimbursements/year; long-term users: $\geq 6$ reimbursements. GC, glucocorticoids; NSAID, non-steroidal anti-inflammatory drug; PPI, proton pump inhibitor. 
representative of the general source population, and the results from studies conducted on the UK medical databases ${ }^{1720}$ seem to be the most comparable with ours. However, direct comparisons are hampered by methodological differences as those studies focused on long-term users. They found a prevalence estimate of about $1 \%$ at any moment, ${ }^{17}{ }^{20}$ a $34 \%$ increase in their use being reported between 1989 and 2008. ${ }^{17}$ Our results regarding the proportion of prevalent users with at least six reimbursements per year $(0.7 \%$ in 2014) are consistent with previous studies on chronic use. Consequently, another cornerstone of our study is that it estimated the overall prevalence of GC use. This highlighted a particularly high use of short-term GC therapy (unique reimbursement, 68\%) that could be specific to France. Regarding the $14 \%$ increase observed in the prevalent use of oral GCs, it is unlikely to be explained only by the expected increase in the annual incidence of their recognised indications in the general population of adults. Moreover, results obtained over a 20-year period in the UK showed that patients newly diagnosed with rheumatoid arthritis or inflammatory bowel diseases are less likely to receive long-term oral GC prescriptions today. ${ }^{17}$

\section{Possible explanations and implications for clinicians and policymakers}

As mentioned above, $68 \%$ of GC initiators received a unique GC reimbursement, most of them being aged less than 50 years. Concurrent use of antibiotics and drugs for respiratory/otological disorders was frequently found at treatment initiation in these individuals, suggesting the presence of underlying ENT or upper respiratory tract infections. Oral GCs are relatively safe for short-term therapy. On the other hand, infections, neuropsychiatric disorders and worsening of pre-existent diabetes are known complications of GC therapy, even in those exposed only for a few days or weeks. The frequent pattern of use found in this study questions the rationale of prescribing oral GCs in adults. For example, firstline therapy for adult chronic sinusitis consists of daily saline irrigation with topical GC therapy. ${ }^{22}$ In this indication, a short course of systemic GC (1-3 weeks) should be considered only in the event of persistent symptoms or acute exacerbation, especially in patients with nasal polyps.

Also worrying was the high prevalence found for comorbidities predisposing to adverse reactions with oral GCs at treatment initiation in long-term users $(25 \%)$. As long-term users were older, a high prevalence of diabetes was expected. Nevertheless, this frequent comorbidity requires attention given the available data showing that diabetes monitoring in long-term GC users is very insufficient. ${ }^{23}$ Adverse psychiatric reactions with GCs are also well known. ${ }^{24}$ Uncontrolled psychotic disorders are a contraindication for GC therapy and the $3.6 \%$ prevalence of psychotic disorders at oral GC initiation found in long-term users is of concern. Moreover, short-term and mid-term users (who represented $98 \%$ of the cohort) are also at risk, as neuropsychiatric symptoms could emerge within a few days or weeks of starting the treatment. ${ }^{25}$

Another key result is the apparently inappropriate prescribing of therapeutic measures associated with GC therapy. The latter is a recognised cause of osteoporosis and osteoporosis management, and DXA measurement should be systematically undertaken in patients whose GC therapy is expected to exceed three consecutive months, especially those at high risk for fractures, including patients aged 70 years and over. ${ }^{2}$ In this study, the use of any drug for osteoporosis management was recorded for fewer than two-thirds of patients at increased risk; in particular only $27 \%$ were prescribed a bisphosphonate. DXA measurement was performed in $13 \%$. This is consistent with previous reports that drugs for osteoporosis management and DXA measurement are used in only a minority of patients exposed to long-term GC therapy. ${ }^{161819}$ Conversely, half of the long-term GC users had concurrent reimbursement of PPIs, apparently without any NSAID or aspirin use, although no consensual recommendation exists regarding the need for such gastric protection. Except in the event of concomitant NSAID use in elderly people, PPIs are advised only if patients have risk factors for peptic ulcer. ${ }^{52627}$ Moreover, inappropriate concurrent use of PPIs in long-term GC users is particularly concerning given that fractures and infection are also associated with PPI use. ${ }^{28}$ Some practitioners prescribe potassium supplementation while others do not. ${ }^{8}$ The present findings suggest that this is infrequent in France except in long-term users (24\%). The latter were more likely to be adequately monitored than short-term and mid-term users, two-thirds having kalaemia monitoring at least once, which is in line with a previous report. $^{23}$

\section{Unanswered questions and future research}

In conclusion, oral GC use is very widespread among adults in France and its prevalence steadily increased over the 2007-2014 period, the overwhelming majority of this being short-term. This could partly be due to an increase in the number of unjustified prescriptions that would exceed the number of those performed in situations where the benefit/risk ratio is recognised favourable. This hypothesis needs to be confirmed by further research and the impact of this extensive use in the population should be estimated. Moreover, our findings plead for the development of interventions designed to improve the monitoring of chronic users with regard to the frequent comorbidities at risk and inappropriate prescribing of preventive therapeutic measures.

Contributors ABL, AP, LF, BB, PN conceptualised and designed the work. EP collected the data and carried out the analysis. ABL, AP, LF, BB, PN interpreted the data. $\mathrm{ABL}$ wrote the first draft. All the authors critically revised and approved the final manuscript.

Funding The present study is part of the Drugs Systematized Assessment in real-liFe Environment (DRUGS-SAFE) research programme funded by the French Medicines Agency (Agence Nationale de Sécurité du Médicament et des Produits de Santé, ANSM; grants received for the year 2016: 950,000 euros). This programme aims at providing an integrated system allowing the concomitant monitoring of drug 
use and safety in France. The potential impact of drugs, frailty of populations and seriousness of risks drive the research programme. This publication represents the views of the authors and does not necessarily represent the opinion of the French Medicines Agency.

Competing interests None declared.

Provenance and peer review Not commissioned; externally peer reviewed.

Data sharing statement № additional data are available.

Open Access This is an Open Access article distributed in accordance with the Creative Commons Attribution Non Commercial (CC BY-NC 4.0) license, which permits others to distribute, remix, adapt, build upon this work non-commercially, and license their derivative works on different terms, provided the original work is properly cited and the use is non-commercial. See: http://creativecommons.org/ licenses/by-nc/4.0/

(c) Article author(s) (or their employer(s) unless otherwise stated in the text of the article) 2017. All rights reserved. No commercial use is permitted unless otherwise expressly granted.

\section{REFERENCES}

1. Chast F. [History of corticotherapy]. Rev Med Interne 2013;34:258-63.

2. Briot K, Cortet B, Roux C, et al. 2014 update of recommendations on the prevention and treatment of glucocorticoid-induced osteoporosis. Joint Bone Spine 2014;81:493-501.

3. Grossman JM, Gordon R, Ranganath VK, et al. American College of Rheumatology 2010 recommendations for the prevention and treatment of glucocorticoid-induced osteoporosis. Arthritis Care Res 2010;62:1515-26.

4. Lekamwasam S, Adachi JD, Agnusdei D, et al. A framework for the development of guidelines for the management of glucocorticoidinduced osteoporosis. Osteoporos Int 2012;23:2257-76.

5. Duru N, van der Goes MC, Jacobs JW, et al. EULAR evidence-based and consensus-based recommendations on the management of medium to high-dose glucocorticoid therapy in rheumatic diseases. Ann Rheum Dis 2013;72:1905-13.

6. van der Goes MC, Jacobs JW, Boers M, et al. Monitoring adverse events of low-dose glucocorticoid therapy: eular recommendations for clinical trials and daily practice. Ann Rheum Dis 2010;69:1913-9.

7. Gennari FJ. Hypokalemia. N Engl J Med 1998;339:451-8.

8. Fardet L, Hanslik T, Blanchon T, et al. [Long-term systemic corticosteroid-therapy associated measures: description of the french internal medicine physicians' practices]. Rev Med Interne 2008;29:975-80.

9. Fardet L, Kassar A, Cabane J, et al. Corticosteroid-induced adverse events in adults: frequency, screening and prevention. Drug Saf 2007;30:861-81.

10. Jones MG, Tsega S, Cho HJ. Inappropriate prescription of Proton pump inhibitors in the setting of steroid use: a teachable moment. JAMA Intern Med 2016;176:594-5.
11. Martínek J, Hlavova K, Zavada F, et al. "A surviving myth"-corticosteroids are still considered ulcerogenic by a majority of physicians. Scand J Gastroenterol 2010;45:1156-61.

12. Bénard-Laribière $A$, Jové $J$, Lassalle $R$, et al. Drug use in french children: a population-based study. Arch Dis Child 2015;100:960-5.

13. Tuppin $P$, de Roquefeuil $L$, Weill $A$, et al. French national health insurance information system and the permanent beneficiaries sample. Rev Epidemiol Sante Publique 2010;58:286-90.

14. Curtis JR, Westfall AO, Allison J, et al. Population-based assessment of adverse events associated with long-term glucocorticoid use. Arthritis Rheum 2006;55:420-6.

15. Fardet L, Petersen I, Nazareth I. Common infections in patients prescribed systemic glucocorticoids in primary care: a PopulationBased Cohort Study. PLoS Med 2016;13:e1002024.

16. Trijau S, de Lamotte G, Pradel V, et al. Osteoporosis prevention among chronic glucocorticoid users: results from a public health insurance database. RMD Open 2016;2:e000249.

17. Fardet L, Petersen I, Nazareth I. Prevalence of long-term oral glucocorticoid prescriptions in the UK over the past 20 years. Rheumatology 2011;50:1982-90.

18. Gudbjornsson B, Juliusson UI, Gudjonsson FV. Prevalence of long term steroid treatment and the frequency of decision making to prevent steroid induced osteoporosis in daily clinical practice. Ann Rheum Dis 2002;61:32-6.

19. Overman RA, Yeh JY, Deal CL. Prevalence of oral glucocorticoid usage in the United States: a general population perspective. Arthritis Care Res 2013;65:294-8.

20. van Staa TP, Leufkens HG, Abenhaim L, et al. Use of oral corticosteroids in the United Kingdom. QJM 2000;93:105-11.

21. Walsh LJ, Wong CA, Pringle M, et al. Use of oral corticosteroids in the community and the prevention of secondary osteoporosis: a cross sectional study. BMJ 1996;313:344-6.

22. Rudmik L, Soler ZM. Medical therapies for adult chronic sinusitis: a systematic review. JAMA 2015;314:926-39.

23. Fardet L, Petersen I, Nazareth I. Monitoring of patients on long-term glucocorticoid therapy: a population-based cohort study. Medicine 2015;94:e647.

24. Patten SB, Neutel Cl. Corticosteroid-induced adverse psychiatric effects: incidence, diagnosis and management. Drug Saf 2000;22:111-22.

25. Fardet L, Petersen I, Nazareth I. Suicidal behavior and severe neuropsychiatric disorders following glucocorticoid therapy in primary care. Am J Psychiatry 2012;169:491-7.

26. O'Mahony D, O'Sullivan D, Byrne S, et al. STOPP/START criteria for potentially inappropriate prescribing in older people: version 2. Age Ageing 2015;44:213-8.

27. Haute Autorité de Santé. Bon usage du médicament. Les inhibiteurs de la pompe à protons chez l'adulte. June 2009. http://www.hassante.fr/portail/upload/docs/application/pdf/2009-06/ipp_adulte_ juin_2009.pdf (accessed 14 December 2016).

28. Schoenfeld AJ, Grady D. Adverse effects associated with Proton pump inhibitors. JAMA Intern Med 2016;176:172-4. 